\title{
Vascular Causes of Dysphonia: A Case Series with Different Etiologies
}

\author{
Pierino Spadafora $\mathbb{D}^{\prime}$ \\ Andrea Esposito $\mathbb{D D}^{2}$ \\ Caterina Giannitto ${ }^{3}$ \\ Letizia Di Meglio' \\ Nunzio Paolo Nuzzi (D) $^{3}$ \\ Gianpaolo Carrafiello ${ }^{2}$ \\ 'Postgraduate School of Diagnostic and \\ Interventional Radiology, University of \\ Milan, Milan, 20122, Italy; ${ }^{2}$ Foundation \\ IRCCS Ca' Granda Maggiore Policlinico \\ Hospital, Radiology Department, Milan, \\ Italy; ${ }^{3}$ Humanitas Research Hospital, \\ Radiology Department, Milan, 200/3, \\ Italy
}

\begin{abstract}
Dysphonia is a frequent and often disabling condition that can be caused by a multitude of circumstances. Differential diagnosis of dysphonia comprehends many different etiologies and many causative agents (neoplasms, inflammations, traumatic injuries) that can occur in a large anatomical space (from the encephalic trunk to the upper mediastinum). It is fundamental to remember that vascular etiologies are responsible for some rare cases of dysphonia. In the radiological database of two urban academic hospitals, from 2012 to 2020, we sought patients who underwent CT or MRI for dysphonia, selecting only the ones with an underlying clinically confirmed vascular etiology. We present three emblematic cases with different vascular etiologies: a ductus arteriosus aneurysm, a left internal carotid artery dilatation, a laryngeal arteriovenous malformation (AVM). Vascular causes of dysphonia are rare, but especially in these cases an accurate and prompt diagnosis is fundamental, in particular considering that the underlying cardiovascular anomaly can often pose a higher risk for the patient than the hoarseness itself. Diagnostic imaging plays a fundamental role in detecting the most common causes of dysphonia but it is very important that radiologists take the vascular causes into account so as not to miss them and to obtain a correct diagnosis.
\end{abstract}

Keywords: dysphonia, thoracic aorta, arteriovenous malformation, carotid artery, patent ductus arteriosus

\section{Introduction}

It is calculated that dysphonia (impaired voice production, characterized by altered vocal quality, loudness or vocal effort) affects nearly one-third of the population at some point in their life and can impair communication or quality of life. ${ }^{1-4}$ Dysphonia can affect patients of all ages, but has a higher prevalence in the elderly, teachers, and other persons with important vocal demands. It is often caused by benign or self-limited conditions, but it may also be the presenting symptom of a more severe and even lifethreatening condition, that requires rapid diagnosis and treatment. ${ }^{4}$

Differential diagnosis of dysphonia comprehends many different etiologies and many causative agents (from neoplasms and inflammations to traumatic injuries) that can occur in a large anatomical space (from the encephalic trunk to the upper mediastinum). ${ }^{5}$

An accurate anatomical study is very important, and it is fundamental to remember that vascular etiologies are responsible for some rare cases of dysphonia.

In the radiological database of two urban academic hospitals, from 2012 to 2020, we sought patients who underwent CT or MRI for dysphonia, selecting only the ones with an underlying clinically confirmed vascular etiology.
Correspondence: Pierino Spadafora Postgraduate School of Diagnostic and Interventional Radiology, University of

Milan, Via Festa del Perdono 7, Milan,

Andrea Esposito

Foundation IRCCS Ca' Granda Maggiore Policlinico Hospital, Radiology

Department, Via F. Sforza 35, Milan,

20122, Italy

Email andrea.esposito@policlinico.mi.it 
We selected three emblematic cases with different etiologies:

- A ductus arteriosus aneurysm;

- A left internal carotid artery dilatation;

- A laryngeal arteriovenous malformation (AVM).

It is very important that radiologists also take into account vascular etiologies when evaluating patients with dysphonia to obtain a correct diagnosis and not to miss these causes.

\section{Case I}

We present the case of a 79-year-old woman complaining of long-term dysphonia, which started about 2 years earlier. A laryngeal endoscopy identified a left vocal cord palsy and a head-neck-chest contrast-enhanced $\mathrm{CT}$ was requested.

According to our hospital protocol, when a vocal cord palsy without any organic lesion is discovered, a headneck-chest $\mathrm{CT}$ with contrast medium is requested by the otorhinolaryngologist to discover any possible extrinsic cause.

Chest CT revealed a large ductus arteriosus aneurysm at the aortic isthmus, partly thrombosed, with a maximum diameter of about $4 \mathrm{~cm}$ (Figure $1 \mathrm{~A}$ and $\mathrm{B}$ ).

Neck CT showed the enlargement of left pyriform sinus and ventricle, medial rotation and thickening of aryepiglottic fold (Figure 1C and D) and abducted ipsilateral arytenoid cartilage, findings compatible with a clinical left vocal cord palsy.

Given the absence of any other organic cause, or involvement of the brain, that could explain this palsy, it was attributed to the compression of the left recurrent laryngeal nerve by the large ductus arteriosus aneurysm.

\section{Case 2}

The second case is represented by a 47-year-old man complaining of dysphonia and dysphagia of sudden onset with laryngeal endoscopic signs of left pharyngeal palsy with right deviated uvula and left vocal cord palsy.

Neurological examinations did not reveal other abnormalities, so the patient, according to our Hospital protocol for vocal cord palsy, as previously described, underwent a head-neck-chest contrast-enhanced CT, which showed a left extracranial internal carotid artery focal dilatation (Figure 2A and B). This dilatation, compressing the vagus nerve, was identified as the cause of the left pharyngeal and laryngeal palsy in this middleaged man.

\section{Case 3}

The third and last case is of a 45-year-old man complaining of dysphonia with laryngeal endoscopic detection of a
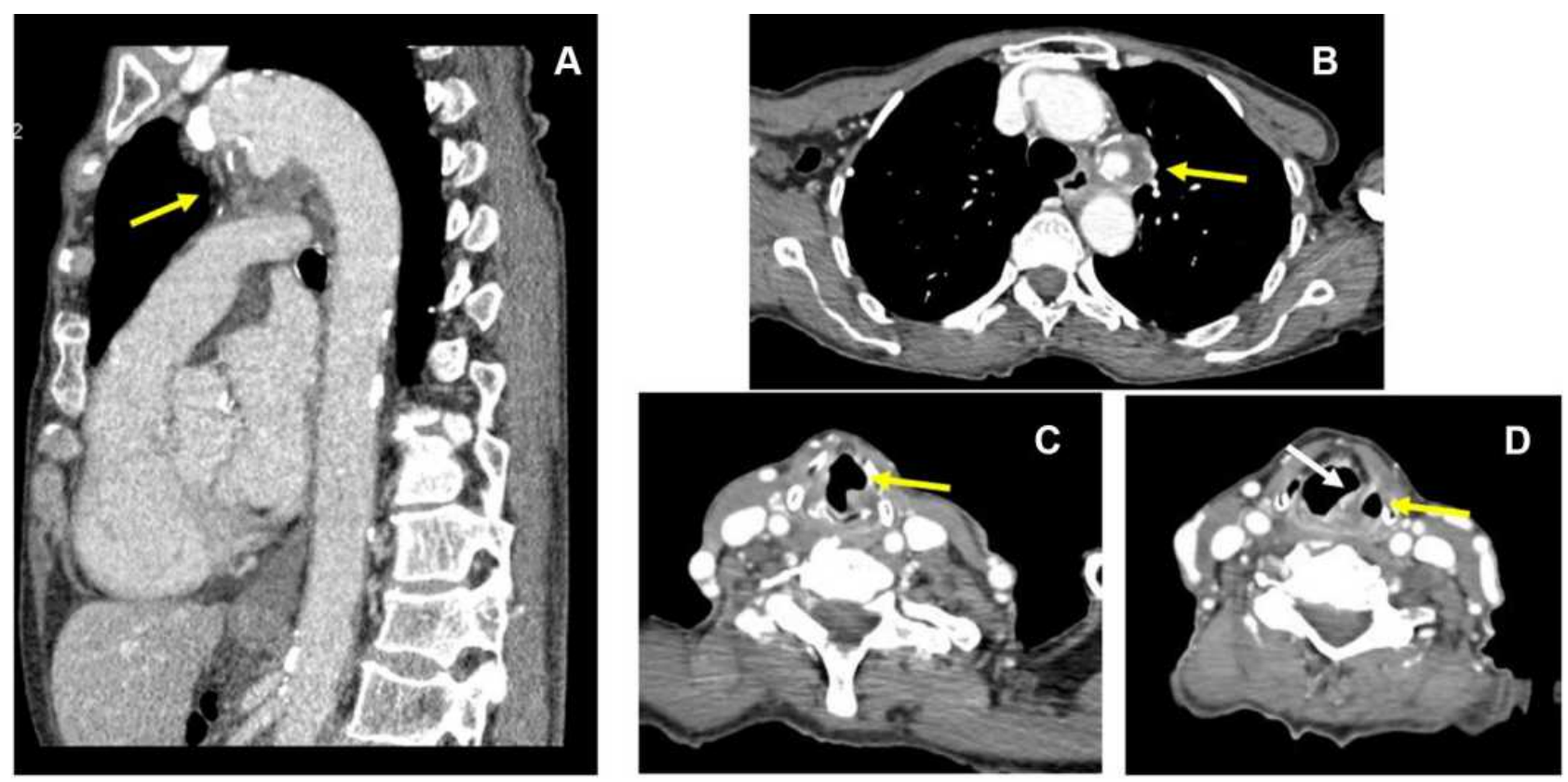

Figure I Ductus bump aneurysm. (A and B) Sagittal reformatted (A) and axial (B) postcontrast CT images of the chest show a ductus bump aneurysm, partly thrombosed (yellow arrows). (C and D) axial postcontrast CT images of the neck show findings compatible with left vocal cord palsy: enlargement of left ventricle (C, yellow arrow) and pyriform sinus (D, yellow arrow), medial rotation and thickening of the left aryepiglottic fold (D, white arrow). 

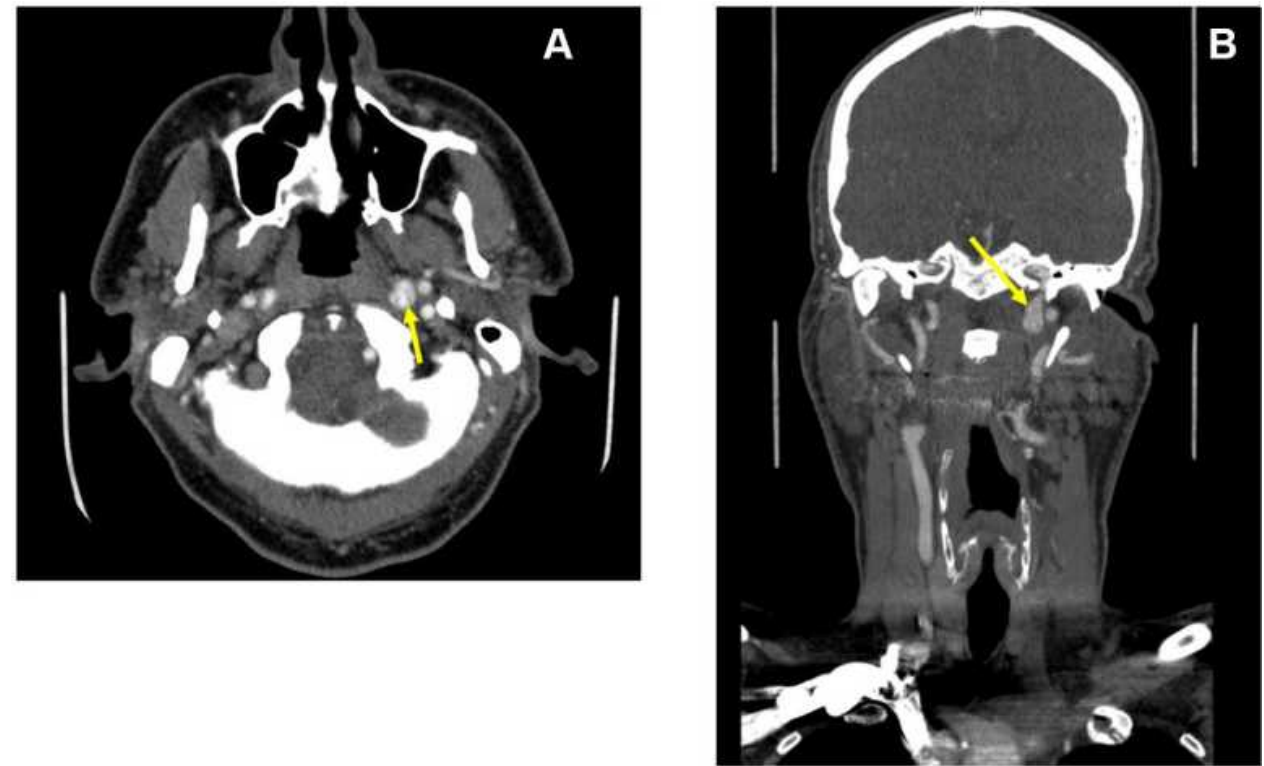

Figure 2 Focal dilatation of left internal carotid artery. (A and B) Axial (A) and coronal reformatted (B) postcontrast CT images show a left extracranial internal carotid artery dilatation (yellow arrows).

round submucosal lump of the left aryepiglottic fold which presented a smooth surface.

As a laryngeal lesion was suspected and to avoid ionizing radiation, the patient underwent a neck MRI, which showed an arteriovenous malformation (AVM) of the left pre-epiglottic space with invasion of the left paraglottic space, at the level of the false vocal cord (Figure $3 \mathrm{~A}-\mathrm{C}$ ). The patient underwent an angiographic procedure, which confirmed an AVM of the left pre-epiglottic and paraglottic space, fed by the left superior thyroid artery (Figure 3D and E). The AVM was treated during the angiography procedure with endovascular embolization.

\section{Discussion}

Dysphonia can originate from a large range of causes, that can develop in a large anatomic area, going from the encephalic trunk to the upper mediastinum. For these reasons, an etiological diagnosis is often difficult.

Dysphonia due to vascular causes is rare and little known, but it is worth considering in the differential diagnosis among the various causes of dysphonia, especially because an accurate and prompt diagnosis is fundamental, considering that the underlying cardiovascular anomaly can often pose a higher risk for the patient than the hoarseness itself. ${ }^{6}$ When there is an underlying cardiovascular anomaly, prognosis depends on this anomaly, and its correction (when possible) is fundamental for the patient's recovery.

In Table 1 vascular causes of dysphonia are listed.

Even though a chest radiograph is usually ordered as the first imaging study for its accessibility and rapidity, a head-neck-chest CT or MRI study should be performed on all patients with recurrent laryngeal nerve palsy, taking into account the many and various possible etiologies. In particular, CT is usually essential in the evaluation of mediastinum, especially of the aortopulmonary region, which can rarely be assessed using a chest radiograph.

Recurrent laryngeal nerve compression by vascular structures is called Ortner's syndrome. The syndrome was described for the first time by Norbert Ortner in 1897 in relation to mitral stenosis with left atrial enlargement, which caused a compression on the left recurrent laryngeal nerve. ${ }^{6-8}$

Nowadays this term includes all the vascular causes of compression on the recurrent laryngeal nerve leading to dysphonia; other causes have been described, such as thoracic aortic aneurysms, aberrant subclavian arteries, 9,10 and an even rarer cause is the compression of the recurrent laryngeal nerve by an aneurysmatic remnant of ductus arteriosus.

Ductus arteriosus aneurysm is a very rare finding in adults. Large ductus arteriosus aneurysms may be incidental CT findings or may present with mass-effect symptoms, as in Case 1 and are associated with life- 

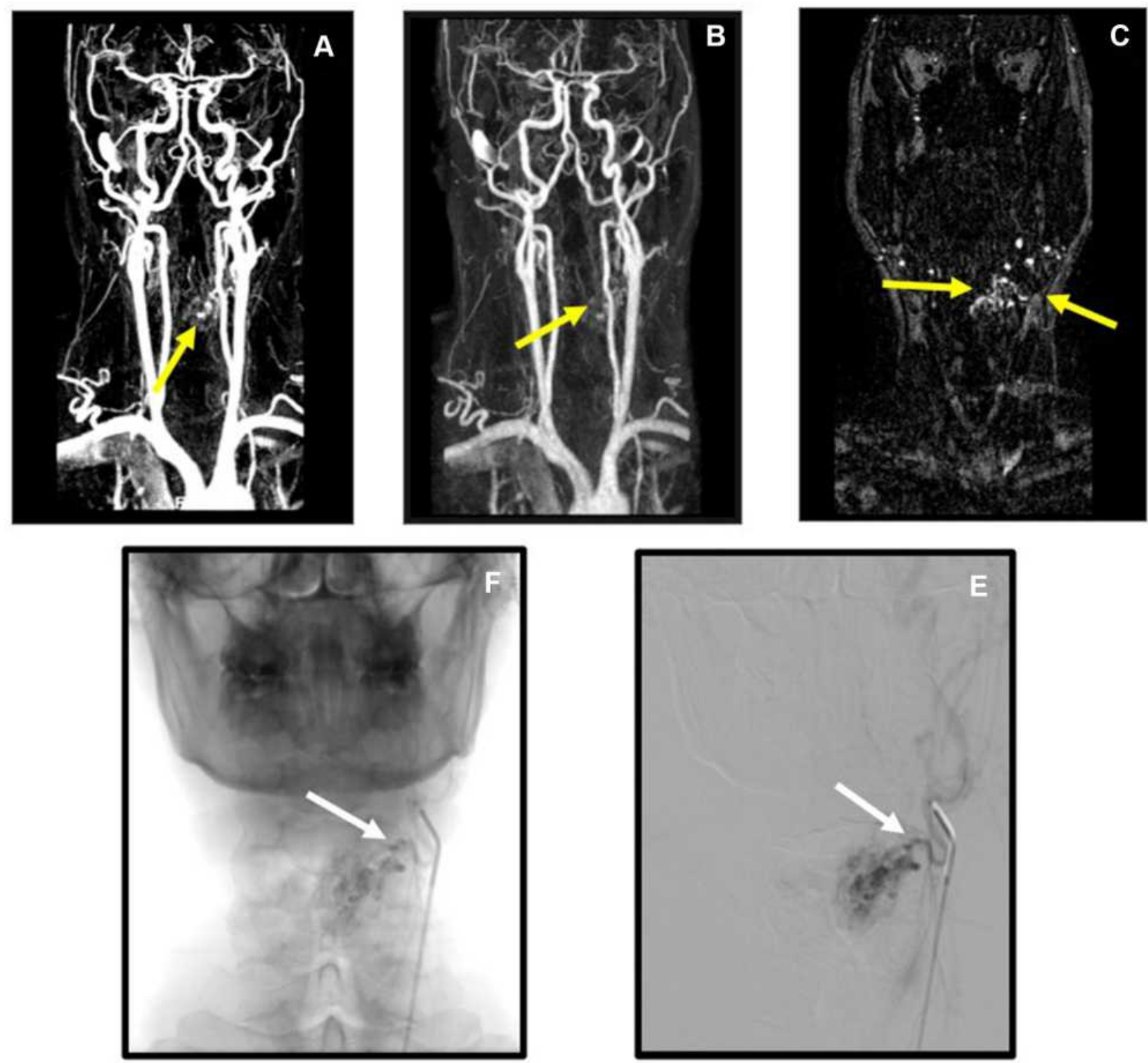

Figure 3 Arteriovenous malformation (AVM) of the left pre-epiglottic and paraglottic space. (A-C) Magnetic Resonance Angiography (MRA) images, with T2 3D fatsaturated sequences, show a AVM of the left paraglottic space at the level of the false vocal cord (yellow arrows). (D and E) Panoramic (D) and selective (E) angiography show that the AVM is fed by the left superior thyroid artery (white arrows).

threatening complications, such as rupture, infection and thromboembolism. ${ }^{11}$

In addition to the aortopulmonary region, the vascular components of the carotid space are also subject to a variety of pathologies.

The carotid space is one of the deep spaces of the neck. It extends from the skull base to the aortic arch and is delimited by the sternocleidomastoid muscle anterolaterally, the visceral space medially and the prevertebral space posteriorly. This anatomic space contains several neurovascular elements, including the carotid artery, the internal jugular vein and the vagus nerve, but it also contains the IX, XI and XII cranial nerves, the sympathetic plexus and deep cervical nodes. Lesions at this level are more often of neurovascular nature, but can arise from any of the described components and, due to the limited space, any expansive or aneurysmatic formation can easily cause compression of the vagus nerve, ${ }^{4,12}$ as in Case 2.

Imaging is often fundamental in the diagnosis of lesions involving the carotid space, whether they be sustained by vascular, inflammatory or malignant causes. 
Table I Vascular Causes of Dysphonia

\begin{tabular}{|l|l|}
\hline Localization & Causes \\
\hline Vocal fold & Vascular lesions (ie arteriovenous malformations) \\
\hline Carotid space & Carotid abnormalities (ie aneurysms/pseudoaneurysms) \\
\hline Mediastinum & $\begin{array}{l}\text { Pulmonary trunk/pulmonary artery enlargement } \\
\text { Aortic aneurysms } \\
\text { Aberrant subclavian arteries } \\
\text { Patent ductus arteriosus aneurysms }\end{array}$ \\
\hline
\end{tabular}

CT and MRI provide important information on the location and extent of the pathology, and they are also fundamental in guiding the choice of the most appropriate therapy.

Cervical AVMs are vascular anomalies. They are usually congenital. Considering that they often reach considerable dimensions, they frequently cause mass effect symptoms, such as dyspnea, dysphagia or dysphonia, depending on their localization.

Intra-operative bleeding is one of the worst surgical complications, due to the presence of vital vascular structures as carotid arteries and jugular veins, and also in the presence of high-flow malformations, which may cause massive bleeding which is difficult to staunch. ${ }^{13-15}$ For this reason, endovascular embolization is an effective therapeutic option as in our third case.

\section{Conclusion}

We presented three cases of dysphonia caused by vascular etiologies where CT and MRI played a fundamental role in detecting them. It is very important that radiologists take these rare causes into account so as not to miss them and to obtain a correct diagnosis.

\section{Ethics Statement}

The patients provided written informed consent for publication of their case reports and accompanying images. This study was conducted in accordance with the Declaration of Helsinki and was accepted by the Ethics Committee of our Institution, Foundation IRCCS Ca' Granda Maggiore Policlinico Hospital.

\section{Disclosure}

The authors report no conflicts of interest in this work.

\section{References}

1. Cohen SM, Kim J, Roy N, Asche C, Courey M. Prevalence and causes of dysphonia in a large treatment-seeking population. Laryngoscope. 2012;122(2):343-348. doi:10.1002/lary.22426

2. Reiter R, Hoffmann TK, Pickhard A, Brosch S. Hoarseness-causes and treatments. Dtsch Arztebl Int. 2015;112(19):329-337. doi:10.3238/arztebl.2015.0329

3. Cohen SM. Self-reported impact of dysphonia in a primary care population. Laryngoscope. 2010;120(10):2022-2032. doi:10.1002/ lary. 21058

4. Stachler RJ, Francis DO, Schwartz SR, et al. Clinical practice guideline: hoarseness (dysphonia) (update) executive summary. Otolaryngol Head Neck Surg. 2018;158(3):409-426. Erratum in: Otolaryngol Head Neck Surg. 2018 Aug;159 (2): 403.PMID: 29494316. doi:10.1177/0194599817751031

5. Montoya S, Portanova A, Bhatt A. A radiologic review of hoarse voice from anatomic and neurologic perspectives. Insights Imaging. 2019;10(1):108. doi:10.1186/s13244-019-0786-7

6. Heikkinen J, Milger K, Alejandre-Lafont E, et al. Cardiovocal syndrome (Ortner's syndrome) associated with chronic thromboembolic pulmonary hypertension and giant pulmonary artery aneurysm: case report and review of the literature. Case Rep Med. 2012;2012:230736. doi:10.1155/2012/230736

7. FitzGerald J, Muller N, Hogg J. A rare cause of Ortner's syndrome (cardiovocal hoarseness). Thorax. 2004;59(7):636. doi:10.1136/ thx.2003.020503

8. Verbeke X, Vliebergh J, Sauer M, Leys M. Hoarseness revealing Ortner's syndrome. Acta Clin Belg. 2015;70(3):230. doi:10.1179/ 2295333715Y.0000000003

9. Hermans C, Manocha S, McLaughlin JE, Lipman M, Lee CA. Ortner syndrome and haemophilia. Haemophilia. 2005;11(5):548-551. doi:10.1111/j.1365-2516.2005.01140.x

10. Escribano JF, Carnes J, Crespo MA, Anton RF. Ortner's syndrome and endoluminal treatment of a thoracic aortic aneurysm: a case report. Vasc Endovascular Surg. 2006;40(1):75-78. doi:10.1177/ 153857440604000111

11. Pontone G, Andreini D, Bartorelli AL, Dainese L, Fusari M, Biglioli P. Incidental detection of a giant ductus arteriosus aneurysm by lowdose multidetector computed tomography in an asymptomatic adult. $J$ Vasc Surg. 2010;51(5):1260-1264. Epub 2010 Jan 18. PMID: 20080001. doi:10.1016/j.jvs.2009.11.051

12. Kuwada C, Mannion K, Aulino JM, Kanekar SG. Imaging of the carotid space. Otolaryngol Clin North Am. 2012;45(6):1273-1292. doi:10.1016/j.otc.2012.08.012

13. Gonzàlez-Garcìa R, Rubio-Correa I, Moreno-Garcìa C. Massive glosso-cervical arteriovenous malformation: the rationale for a challenging surgical resection. J Clin Exp Dent. 2014;6(4):e456-e459. doi: $10.4317 /$ jced.51608 
14. Kobayashi K, Nakao K, Kishishita S, et al. Vascular malformations of the head and neck. Auris Nasus Larynx. 2013;40(1):89-92. doi:10.1016/j.anl.2012.02.002
15. Rutt A, Ozgursoy SK, Paz-Fumagalli R. Laryngeal venous malformation. Ear Nose Throat J. 2020;99(6):367-368. doi:10.1177/ 0145561319840136

\section{Publish your work in this journal}

Reports in Medical Imaging is an international, peer-reviewed, open access journal publishing original research, reports, reviews and commentaries on all areas of medical imaging. The manuscript management

Submit your manuscript here: https://www.dovepress.com/reports-in-medical-imaging-journal system is completely online and includes a very quick and fair peerreview system, which is all easy to use. Visit http://www.dovepress. com/testimonials.php to read real quotes from published authors. 